\title{
ARTÍCULOS
}

\section{AÑOS DE AUTOMATIZACIÓN DE PRÉSTAMO INTERBIBLIOTECARIO EN ESPAÑA (1992-2012)}

\author{
Josep-Manuel Rodríguez-Gairín
}

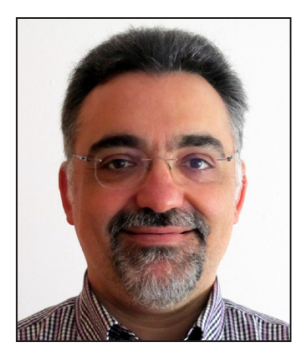

Josep-Manuel Rodríguez-Gairín es profesor titular de la Facultat de Biblioteconomia i Documentació de la Universitat de Barcelona, donde también coordina las aulas de informática y asesora en aspectos tecnológicos. Ha llevado a cabo la infraestructura de proyectos como Revistas digitales de biblioteconomía y documentación (Temaria), revista BiD: Textos universitaris de biblioteconomia i documentació, Matriz de información para la evaluación de revistas (Miar), Recursos en línia per elaborar treballs de recerca (Alehoop), Directorio de expertos en el tratamiento de la información (EXIT), International registry of authors-Links to identify scientists (IraLIS), etc. Es miembro de Ciepi, ThinkEPI y del consejo técnico del repositorio E-LIS. Fundador de la empresa Kronosdoc, dedicada a la asesoría y desarrollo de programas de gestión documental.

http://orcid.org/0000-0001-8375-7911

Universitat de Barcelona Facultat de Biblioteconomia i Documentació Melcior de Palau, 140. 08014 Barcelona rodriguez.gairin@ub.edu

\section{Resumen}

Se presenta la evolución en los últimos veinte años (1992-2012) de la automatización del préstamo interbibliotecario en las bibliotecas universitarias españolas. A partir de los distintos procesos que se llevan a cabo en este servicio-envío de peticiones, localización y recepción de documentos y gestión interna-, se identifica cada uno de los programas utilizados y se pone de manifiesto como han evolucionado cada uno de los procedimientos en paralelo a la propia tecnología.

\section{Palabras clave}

Préstamo interbibliotecario, Obtención de documentos, Automatización, Bibliotecas universitarias, España.

\section{Title: Twenty years of interlibrary lending and document supply automation in Spanish academic libraries} (1992-2012)

\begin{abstract}
Trends over the past 20 years (1992-2012) of interlibrary lending and document supply (ILDS) automation in Spanish academic libraries are discussed. Taking into account the routines and procedures carried out by ILDS services -document identification, localization, document request, reception of borrowed items and user services or internal management- the article lists the software used in Spanish universities to handle these services, setting it into the context of the evolution of each process in parallel with software developments.
\end{abstract}

\section{Keywords}

Interlibrary loan, Document delivery, Automation, Academic libraries, Spain.

Rodríguez-Gairín, Josep-Manuel. "20 años de automatización de préstamo interbibliotecario en España (1992-2012)”. El profesional de la información, 2012, noviembre-diciembre, v. 21, n. 6, pp. 557-566.

http://dx.doi.org/10.3145/epi.2012.nov.02

\section{Introducción}

En 1992 un grupo de bibliotecas Rebiun: las de las universidades Rovira i Virgili, Barcelona, Carlos III de Madrid, Canta- bria, Alcalá de Henares, País Vasco, Nacional de Educación a Distancia y Santiago de Compostela adquieren de forma consorciada el programa $S O D$ para la gestión del préstamo interbibliotecario (en adelante, PI) que estaba creando en 
esos momentos la Universitat Politècnica de Catalunya. Era un año en el que las nuevas tecnologías empezaban a introducirse de forma incipiente en las bibliotecas. Desde entonces la tecnología ha avanzado muchísimo y el PI ha ido adaptando sus tareas siguiendo esos avances

En este artículo se revisan los programas utilizados en los procesos de $\mathrm{Pl}$, principalmente en las universidades españolas. Algunos han desaparecido por el avance tecnológico, y otros han persistido adecuándose a los cambios.

\section{0 años de automatización del PI}

La década de los 90 fue una época de crecimiento en los servicios de PI. Urbano (1997) destaca cuatro razones fundamentales:

- renovado interés por la cooperación interbibliotecaria;

- mayor facilidad de identificación y localización de documentos;

- dificultades de obtención rápida de los documentos externos a la colección;

- necesidad de definir nuevos modelos de trabajo bibliotecario compatibles con las nuevas formas de edición y distribución electrónica.

A nivel internacional encontramos mucha bibliografía que refleja los cambios que la automatización provocaba en estos servicios (Leeves, 1993; Nagelsmeier-Linke, 1994; Lieberthal, 2000; Porat, 2001) y su influencia en otros procesos como la mejora de las colecciones (Bartolo, 1989). El desarrollo de las normas ISO 10160 y 10161 (1997), el protocolo OpenURL (NISO Z39.88, 2004) y las recomendaciones de la IFLA en materia de PI (Stein, 2007) contribuyeron a facilitar la interconexión de los diferentes programas aunque, en la práctica, a día de hoy aún no están completamente aplicadas.

En España los datos recogidos por Rebiun en sus anuarios demuestran que el servicio ha incrementado en estas dos últimas décadas el número total de peticiones, tanto de solicitudes como de suministros, pasando de 346.351 en 1994 a 390.135 en 2010. En el gráfico 1 se observa una tendencia a la estabilización en los últimos años que se puede atribuir

\footnotetext{
-Todas las peticiones deberían tramitarse en un sistema electrónico, que, además, fuera capaz de interactuar con otros sistemas de préstamo interbibliotecario.

-Aconseje a las bibliotecas que envíen sus peticiones electrónicamente

-De a los usuarios finales la posibilidad de comprobar en línea el estado de sus peticiones

-Comunique electrónicamente el estado de las peticiones

-Introduzca nuevas tecnologías en todos los procesos

-Envíe electrónicamente las reproducciones si es posible

-Implemente formularios de petición en línea y/o que puedan interactuar con otros sistemas de suministro de documentos
}

Tabla 1. Principales recomendaciones IFLA que afectan a la automatización del PI a la mejora en las colecciones en papel, al incremento de las electrónicas -compradas por los centros o consorciadamente- $y$, por descontado, a los efectos de la crisis económica. Es interesante remarcar que estos factores no han provocado un importante descenso en el número de peticiones, como cabría esperar, lo que nos lleva a pensar que existen otros factores que condicionan la persistencia del servicio.

En términos de automatización, una encuesta realizada en 1994 por la Asociación Española de Archiveros, Bibliotecarios, Museólogos y Documentalistas de España (Anabad) revelaba que las bibliotecas universitarias eran las que en mayor proporción, un $76 \%$, tenían gestión automática del $\mathrm{PI}$, el 64\% de ellas con el programa SOD (López-Manzanedo; Vázquez-Valero; Goas-Paz, 1996, p. 80).

\section{El incremento de colecciones electróni- cas en las bibliotecas no ha provocado un descenso importante en el número de peticiones de acceso al documento}

Los servicios de PI realizaban las peticiones a sus suministradores usando el correo postal y, en caso de urgencia, por medio del teléfono o del fax, aunque este último aún era considerado un lujo en una gran parte de ellos.

\section{Automatización de comunicaciones y procesos}

Para sistematizar la exposición de las distintas aplicaciones implicadas en el PI, estableceremos cinco tipos de procesos susceptibles de ser automatizados:

- envío de las peticiones;

- envío/recepción de los documentos digitalizados;

- localización del documento;

- acceso del usuario al servicio;

- procesos internos de control del estado de las peticiones, reclamaciones, gestión administrativa y obtención de estadísticas.

\section{Envío de peticiones: de Crosstalk a Outlook}

La automatización del PI se inicia en blanco y negro, o mejor dicho en blanco y verde, cuando a principios de los años 80 los terminales VT100 de Digital Equipment Corporation utilizaban fósforo de tipo P1 para mostrar los caracteres ascii. Poco después llegaron los primeros ordenadores personales IBM a nuestras bibliotecas y, aunque empleaban fósforo P4 blanco, aún faltaban algunos años para que el Windows 3.11 llenara de color nuestras pantallas.

En esa época tampoco existía internet tal como la conocemos hoy. Los computadores se interconectaban usando la red telefónica convencional por medio de un acoplador acústico, un aparato que convertía la señal telefónica en señal digital y la transmitía a la velocidad de 300 bauds -300 bits por segundo. Su sucesor, el módem, supuso un gran avance al multiplicar esa velocidad por cuatro aunque aún estaba muy lejos de los $100 \mathrm{Mbps}-100$ millones de bits por segundo-a los que nos empiezan a acostumbrar las compañías telefónicas actuales. 


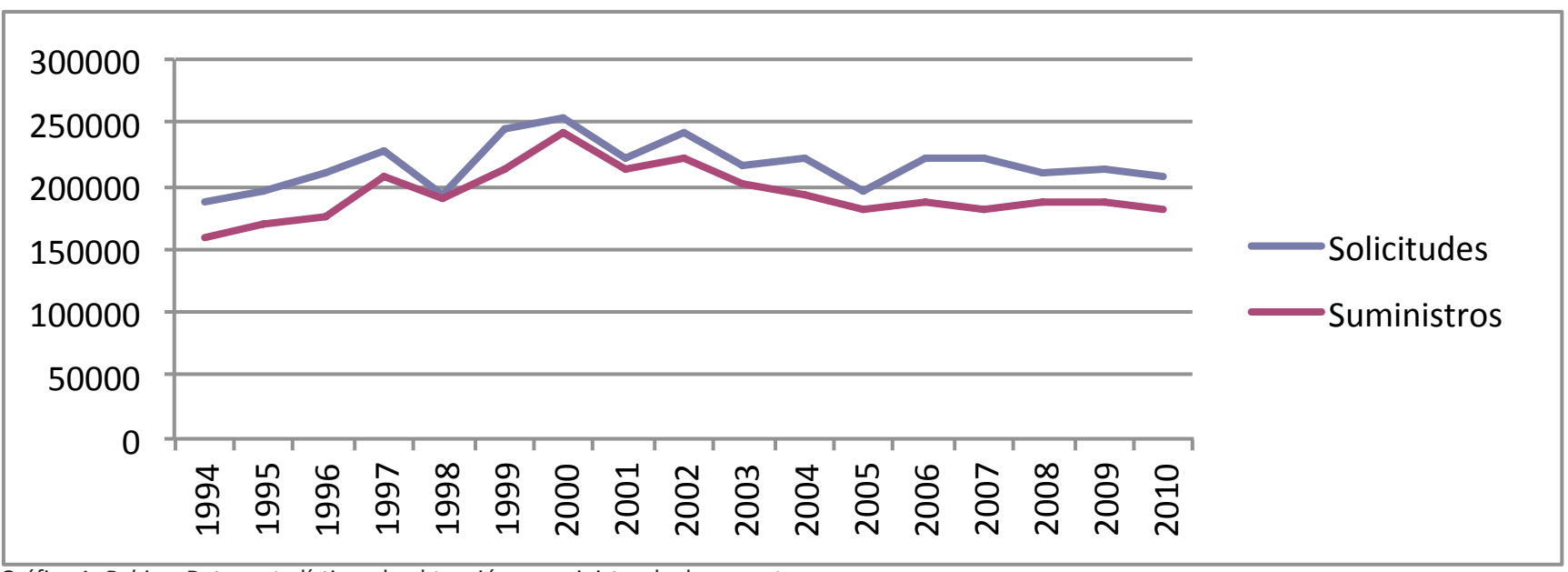

Gráfico 1. Rebiun. Datos estadísticos de obtención y suministro de documentos

http://estadisticas.rebiun.org/cuestionarios/indicadores/indicadores_main.asp\#

Si bien la conexión se podía establecer directamente entre los dos centros por la red telefónica conmutada, lo más adecuado era usar Iberpac, la red de datos española basada en el protocolo X.25, a la que se podía conectar desde la red convencional a través de unos números de tres cifras utilizando el protocolo X.28 (Baiget, 1992). El uso de Iberpac se consideraba una llamada local, por lo que se abarataba el coste de la comunicación aunque el destinatario estuviera al otro lado del Atlántico. Al tratarse de una señal digital, la calidad de la transmisión estaba libre de ruidos y se evitaban los cortes e interferencias de la comunicación directa.

\section{ARTTel}

A principios de los años 90, algunas bibliotecas empiezan a utilizar ARTTel, un servicio para el envío de peticiones al British Library Document Supply Centre, el principal suministrador internacional. La biblioteca debía solicitar un código de usuario y contraseña y conectarse al sistema llamando directamente a un teléfono del Reino Unido, a través de Iberpac o, con la aparición de internet, vía telnet. Una vez conectado, el usuario introducía su código, la contraseña, cuatro líneas en blanco, una línea empezando por TX y el número de petición y hasta 11 líneas con los datos bibliográficos en un formato concreto. Se podían separar varias peticiones con cuatro líneas en blanco y se finalizaba con una línea con el texto NNNN. Todo este proceso podía automatizarse usando programas como Crosstalk XVI o ProComm. Independientemente del buen servicio que proporcionaba la British Library, el uso de este sistema automatizado eliminaba la incertidumbre en la recepción de la petición que hasta entonces provocaba el correo ordinario ya que el propio sistema notificaba la correcta llegada.

Con la aparición de internet y del correo electrónico, este sistema se complementó con ARTEmail, que hace posible enviar el mismo formato a una dirección de correo electrónico y con $A R T W e b$, un formulario web en el que el usuario o centro introduce directamente los datos bibliográficos de las peticiones.

http://www.bl.uk/reshelp/atyourdesk/docsupply/index. html

ARTTel fue clausurado el 31 de enero del 2011, pero ARTEmail y $A R T W e b$ continúan activos y los programas de gestión que describiremos más adelante incorporan sistemas para dar el formato correcto y enviar la petición sin necesidad de volver a teclear los datos bibliográficos.

En la actualidad el uso del correo electrónico junto a los formularios web se han consolidado como los dos mecanismos más utilizados para realizar peticiones (Duarte; Olivé, 1996). El segundo, a diferencia del correo, también elimina la incertidumbre en la recepción. Para evitar volver a teclear los datos en el sistema de gestión, la IFLA propuso una serie de recomendaciones sobre los datos a incluir y formato a emplear (Gould, 2000) instando también a adoptar las normas ISO 10160/10161. Muchas bases de datos y catálogos usan protocolos como el OpenURL para trasladar la información bibliográfica directamente a los formularios de los programas de gestión.

Los formularios web como mecanismo de introducción de peticiones directamente al sistema de automatización están presentes en todos los programas de PI actuales aunque también se utilizaron como pasarela para formatear los datos bibliográficos y enviarlos por correo electrónico al servicio, como es el caso de Sad@Exe elaborado en la Universidad de Jaén (Navarrete-Cortés; Navarrete-Cortés, 2000).

\section{Envío de documentos: del fax a los servicios web}

Hasta la aparición del fax, tanto el envío de originales en préstamo como el de copias de artículos se realizaban por correo ordinario y eran habituales demoras de 7 a 14 días o más. El fax facilita la recepción de la copia de un artículo de cualquier biblioteca del mundo de manera instantánea aunque en la práctica, un desfase de unas 24 horas era considerado aceptable por los diferentes horarios o la demora en su preparación. Muchas pequeñas bibliotecas eran reticentes a enviar los artículos por fax ya que implicaba fotocopiarlos previamente y pasarlos manual y lentamente en aparatos que carecían de alimentador de hojas. Así mismo, las bibliotecas solicitantes tenían que asumir el coste elevado de la transmisión telefónica y en la mayoría de casos la calidad del documento recibido, por supuesto en blanco y negro y papel térmico, era bastante pésima, sobre todo si incluía fotografías. Por estas razones el fax se empleaba únicamente en casos de necesidad urgente. 


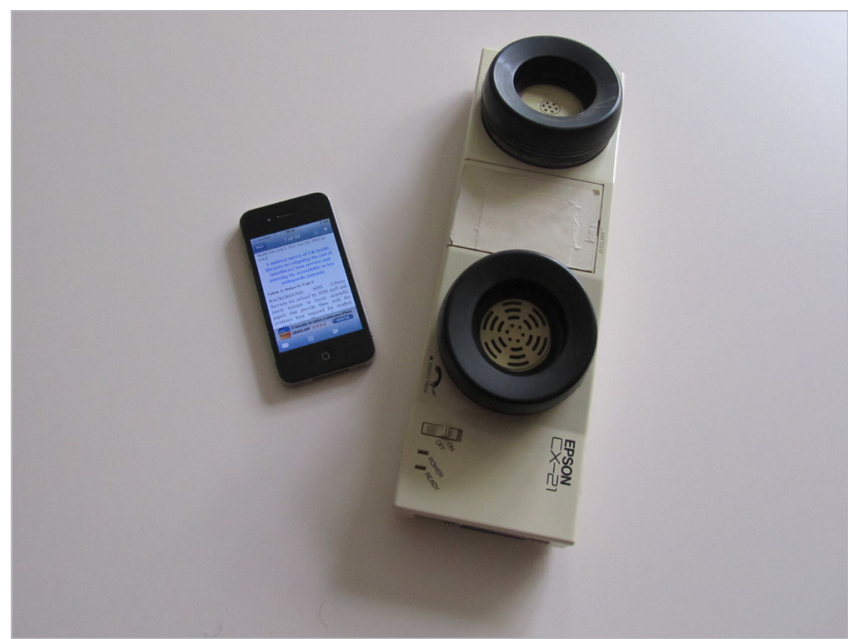

Figura 1. Del acoplador acústico a los teléfonos inteligentes

\section{Ariel}

Es una aplicación creada en 1990 por RLG, The Research Library Group Inc., que surge como una alternativa al fax, efectiva y de bajo coste (Landes, 1997). Ariel emplea internet a través del protocolo FTP para transmitir el documento desde la biblioteca suministradora a la peticionaria, lo que supone un gran ahorro económico respecto al fax. Sin embargo precisaba un coste inicial importante de hardware para asegurar una gran calidad ya que se recomendaba disponer de una impresora láser y de un escáner. En sus primeras versiones Ariel funcionaba en un entorno DOS lo que obligaba a disponer de un ordenador dedicado, conectado continuamente y pendiente de la recepción. Con la introducción de la versión Windows, podía activarse en segundo plano de manera que el mismo ordenador se utilizaba para otras tareas en la biblioteca. Otro ahorro importante respecto al fax era que el documento no necesitaba ser fotocopiado previamente sino que se escaneaba directamente del original.

Ariel empleaba el formato TIFF multipágina y requería programas específicos para su visualización por lo que la mayoría de bibliotecas optaban por imprimir el documento recibido y entregarlo en papel al solicitante.

Ariel fue comprado por Infotrieve en 2003 y la empresa que lo diseñó, RLG, se fusionó con OCLC en 2009. El uso de Ariel se extendió entre las bibliotecas españolas, especialmente en el desaparecido Cindoc (Centro de Información y Documentación Científica) (Ramos; Montes, 1998) y en las bibliotecas universitarias.

http://www.infotrieve.com/ariel-interlibrary-loan-software

\section{Prospero}

En la última obra de Shakespeare -La tempestad- el mago Prospero, abandonado a su suerte en una isla tras naufragar su barco, utiliza al espíritu Ariel, al que sólo ve él, para alcanzar sus objetivos. Emulando esta obra, Prospero es una aplicación que oculta al administrador los documentos recibidos por Ariel y en primer lugar los transforma en pdf. A partir de aquí consulta una base de datos de usuarios para decidir si el documento debe ser almacenado para su impresión o si el usuario ha aportado una dirección de correo electrónico en cuyo caso le envía un mensaje con un enlace para su descarga. Es un programa de código abierto y gratuito creado en 1999 por la biblioteca de la Ohio University que podemos considerar un complemento a Ariel (Schnell, 2000) ya que suple sus dos principales carencias: convertir los documentos a pdf y hacer posible que el usuario pueda descargarlos directamente.

http://bones.med.ohio-state.edu/prospero

En España también se utilizó Prospero en varias universidades como es el caso del Centro de Documentación Científica de la Universidad de Zaragoza (Angós-Ullate; FernándezRuiz; Salvador-Oliván, 2003).

\section{DocView y DocMorph}

Aunque su utilización en las bibliotecas universitarias españolas ha sido prácticamente nula, merece la pena reseñar estas dos aplicaciones creadas por la National Library of Medicine como alternativa al sistema Ariel.

- DocView incorpora un visualizador de formato tiff, pudiendo utilizar los documentos generados por Ariel, así como un sistema de transmisión basado en ftp.

http://docmorph.nlm.nih.gov/docview

- DocMorph era un servicio gratuito vía web que transformaba los documentos tiff en pdf y los hacía accesibles al usuario en unas horas.

http://docmorph.nlm.nih.gov/docmorph

Aunque el correo electrónico es fiable, tiene el inconveniente de no disponer de la certeza en la recepción del mensaje ya que no existe un estándar de acuse de recibo

\section{Correo electrónico / descarga web}

Con la generalización de internet el correo electrónico ha pasado a ser el principal sistema para el envío de documentos en formato digital. Si bien lo habitual es enviar el documento como adjunto a un mensaje, esta opción puede presentar problemas en caso de ficheros de gran tamaño ya que muchos servidores de correo tienen limitaciones al respecto y también pueden aparecer conflictos con filtros antispam. Una alternativa muy utilizada es enviar por correo electrónico un enlace que apunte a una web donde el usuario puede descargar el documento. Generalmente esta descarga está protegida por un código de usuario y contraseña y tiene una caducidad de días. Aunque la mensajería electrónica es muy fiable, su principal inconveniente es no disponer de la certeza en la recepción del mensaje ya que no existe un estándar de acuse de recibo en los clientes de correo utilizados.

\section{Servicios web}

Son un conjunto de tecnologías basadas en lenguajes como $\mathrm{xml}$ y protocolos de comunicación como SOAP (simple object access protocol) o XML-RPC ( $x m /$ remote procedure call), que interconectan dos aplicaciones y facilitan el intercambio 
directo de datos. Las ventajas de su utilización en el PI ya han sido reconocidas por varios autores (Méndez-Martínez; Ruz-Baños; Aldehuela-Serra, 2004) aunque en la actualidad sólo algunas aplicaciones como GTBib-SOD emplean este tipo de tecnologías para enviar, tanto las peticiones como los documentos, desde la aplicación de una universidad a otra sin necesidad de usar el correo electrónico ni disponer de servidores de FTP ni programas como Ariel. Los servicios web dan al usuario la certeza de que el documento se ha recibido y no presentan problemas con filtros antispam ni con el tamaño del documento por lo que suponen una buena alternativa al correo electrónico.

\section{Localización del documento: del catálogo a las herramientas de descubrimiento}

\section{Catálogos colectivos}

Hablar de mejoras en la localización de documentos es hablar de cooperación bibliotecaria y catálogos colectivos, un tema del que existe mucha literatura (Casares et al., 1997; Merlo-Vega; Sorli-Rojo, 1998; Merlo-Vega, 1999; Anglada, 2007). La necesidad de un sistema nacional de préstamo en España fue puesta ya de manifiesto en la década de los 80 (Vázquez-Valero; Román-Román, 1986). Se planteaban dos opciones, crear una gran biblioteca de préstamo usando el modelo de la British Library Document Supply Center o planificar un sistema descentralizado y coordinado. Esta segunda opción fue la elegida y se plasmó en la creación del catálogo colectivo de la red de bibliotecas del CSIC, el catálogo colectivo de la red de bibliotecas universitarias Rebiun y el catálogo de la Biblioteca Nacional de España. Con posterioridad se han creado otros catálogos colectivos en comunidades autónomas, temáticos o en bibliotecas públicas.

Hablando estrictamente de automatización y centrándonos en publicaciones periódicas, este autor considera que en el caso de las universidades, los catálogos colectivos presentan una gran deficiencia: la falta de uniformidad de los fondos de revistas que dificulta automatizar la localización de un año o volumen. En muchos casos, como por ejemplo Rebiun, no incluyen esos campos sino que remiten al catálogo local correspondiente.

En contrapartida, merece la pena destacar la buena organización de catálogos temáticos como el C17 (Catálogo de publicaciones periódicas en bibliotecas de ciencias de la salud españolas), Catálogo colectivo de la Coordinadora de Documentación Biomédica $(C D B)$ de la que sólo se publicó la versión en papel, Mecano (Catálogo colectivo de hemerotecas españolas de ingeniería, informática y tecnología) sin continuidad actual, o Documat (Catálogo colectivo de publicaciones periódicas del área de matemáticas), reconvertido en una base de sumarios alojada en Dialnet.

http://rebiun.org/Catalogo

http://www.c17.net

http://documat.unirioja.es

Entre éstos destacamos en términos de automatización C17, que dispone de una interfaz $\mathrm{xml}$ que ha facilitado que los sistemas de automatización de PI desplieguen pasarelas REST para la localización automática de posibles ubicaciones de un año concreto de una publicación. En la actualidad tanto el catálogo como el uso de esta pasarela son de acceso restringido bajo subscripción.

http://es.wikipedia.org/wiki/Representational_State_ Transfer

Se ha de reconocer la importancia que ha supuesto en el PI disponer de catálogos que centralicen las colecciones. En el caso de Rebiun, la incorporación de un servidor que usa el protocolo Z39.50 (1998) - ISO 23950- ha facilitado la automatización de la localización de monografías mediante agentes (Rodríguez-Gairín; Somoza-Fernández, 2005)

Los catálogos colectivos presentan una gran deficiencia: falta de uniformidad de los fondos de revistas que dificulta automatizar la localización de un año o volumen

\section{UnCover}

En la mayoría de casos la rápida localización de artículos de revistas implica disponer de unos datos bibliográficos completos y correctos. Por esta razón los servicios de PI realizan consultas previas a bases de datos con la finalidad de asegurar que el año, volumen o número de páginas sea correcto. Con excepción de Medline, la mayor parte de bases de datos bibliográficas eran de acceso restringido bajo subscripción hasta la aparición de las bases de datos de sumarios que, emulando a Current Contents, recogen el vaciado de cada publicación periódica.

En una revisión histórica, es obligado mencionar UnCover, un proyecto de la Colorado Alliance of Reseach Libraries (CARL) iniciado en 1988 que daba acceso al contenido de más de 500.000 artículos de unas 7.000 revistas disponibles en las bibliotecas de este consorcio (Jaramillo; Squire, 1990). Gracias a UnCover, los servicios de obtención de documentos no sólo completaban las referencias bibliográficas sino que podían solicitar los documentos a cualquiera de esas bibliotecas consorciadas. Es el antecesor de los boletines de sumarios que muchas bibliotecas universitarias españolas iniciaron en los 90 como Compludoc en la Universidad Complutense de Madrid, Winsumar en la Universitat Politécnica de Catalunya o la base de datos de sumarios del CBUC entre otras (Rodríguez-Gairín, 2001; Merlo Vega; Sorli Rojo, 2002).

\section{Adonis}

La gran revolución en este punto es el acceso directo al documento electrónico. Pudiera parecer que este aspecto es relativamente nuevo pero las editoriales ya contemplaron esta posibilidad en los inicios de los soportes ópticos, una tecnología capaz de almacenar gran cantidad de información y susceptible por tanto de incluir las imágenes digitalizadas de los artículos de revistas. Un ejemplo es el proyecto Adonis.

Fue un proyecto piloto elaborado por la British Library Lending Division y la editorial Elsevier entre 1987 y 1988 que 


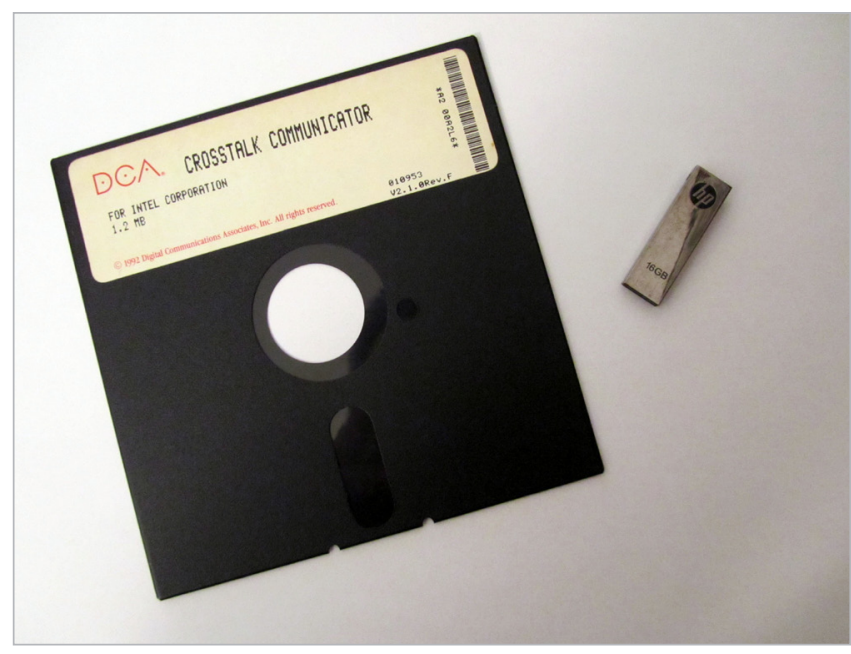

Figura 2. 20 años de Crosstalk. El programa se suministraba en un disquete (floppy disk) de 5,25". A la derecha vemos un pen drive actual con una capacidad dos mil veces superior.

distribuía artículos de 219 revistas, mayoritariamente del ámbito de ciencias de la salud (Merry, 1988). Su gran innovación es que el programa de gestión controlaba los artículos impresos y facilitaba el pago por artículo al editor. Sin embargo los elevados costes de producción y de infraestructura no favorecían su distribución generalizada por lo que Adonis contempló que sólo un centro de cada país de los que participaron tuviera el sistema, y en el caso de España fue el CSIC.

\section{SFX y metabuscadores}

SFX es un "resolvedor" de enlaces basado en el protocolo OpenURL creado por la empresa EXLibris. Es utilizado por varias universidades españolas a título individual así como por consorcios como el CBUC (Consorci de biblioteques universitàries de Catalunya) y el CBUG (Consorcio de bibliotecas universitarias de Galicia). Una relación detallada puede encontrarse en la web del grupo de usuarios de productos EXLibris - Expania. El uso de tecnología OpenURL ha facilitado que las aplicaciones de PI desplieguen conectores que, por ejemplo, pueden advertir al usuario que está solicitando un artículo que dicho documento se encuentra disponible en su institución y por tanto no necesita solicitarlo externamente.

http://www.exlibrisgroup.com/category/SFXOverview http://www.expania.es

Como complemento a SFX nacen metabuscadores como MetaLib o Zportal, aplicaciones que realizan búsquedas secuenciales en distintos recursos y los presentan de manera unificada al usuario.

http://www.exlibrisgroup.com/category/MetaLibOverview http://www.oclc.org/zportal/default.htm

\section{Herramientas de descubrimiento}

Los catálogos colectivos y los metabuscadores están dando paso en los últimos años a las denominadas "herramientas de descubrimiento" -discovery tools - (Breeding, 2012). Se caracterizan por su simplicidad, emulando la caja de búsqueda de Google, y permiten integrar en un índice único resultados de fondos locales, como el catálogo con resultados de productos licenciados disponibles en la Red o de acceso abierto. Luther y Kelly (2011) han realizado un estudio detallado sobre este tipo de aplicaciones y destacan cuatro:

- OCLC WorldCat Discovery Services. Está siendo implementado en universidades como la Universidad de Alicante o el Consorcio de Bibliotecas Universitarias de Castilla y León (Bucle).

http://www.oclc.org/services/reference/default.htm

- Summon. Herramienta de la empresa Serials Solutions, escogida para el desarrollo de la nueva Biblioteca Virtual del Consejo Superior de Investigaciones Científicas (CSIC). http://www.serialssolutions.com/en/services/summon

- Ebsco Discovery Service.

http://www.ebscohost.com/international/default.php?p ar=2\&id=279\&language $=$ spanish

- Primo Central, Discovery and Delivery. Producido por la empresa ExLibris y distribuido en España por GreenData. http://greendata.es/software-de-gestion-de-bibliotecas/ primo/primo-central

\section{Acercamiento del servicio al usuario}

Si en algún punto la automatización del servicio de PI ha supuesto un cambio revolucionario es en la posibilidad de acercar el servicio al usuario. Hasta la aparición del correo electrónico, tanto el usuario como la biblioteca solicitante realizaban la petición en papel, entregándola personalmente o enviándola por correo ordinario y no volvían a tener ninguna información de la misma hasta que recibían el documento. Sólo en casos excepcionales se comprobaba telefónicamente la recepción y las reclamaciones estaban sujetas a la espera de un tiempo prudencial con la incertidumbre de si se habría recibido. En el caso de no disponer del documento, las bibliotecas suministradoras debían notificarlo pero eso no siempre se hacía.

Lo más interesante es la posibilidad de estar continuamente informado del estado de la petición y realizar solicitudes o reclamaciones desde la web

Con la aparición del correo electrónico y de la Web, el usuario teclea directamente los datos en un formulario que se remite por correo electrónico o, mucho mejor, se inserta directamente en el sistema de gestión asegurando la recepción. El servicio no tiene que volver a introducir los datos bibliográficos e incluso la aplicación los completa automáticamente a partir del DOI o de otros identificadores como el PMID de Medline.

Pero sin duda lo más interesante para el usuario o la biblioteca solicitante es la posibilidad de estar continuamente informado del estado de su petición, ya sea porque reciba automáticamente un email cuando cambia el estado -se tramita, se recibe una respuesta negativa, se reclama- o bien porque disponga de una interfaz web personalizada donde poder consultar sus peticiones, descargar los documentos electrónicos recibidos o realizar reclamaciones o cualquier tipo de consultas. 


\section{Automatización de los procesos internos del servicio}

La automatización del servicio de PI surge como necesidad de llevar un control de las peticiones recibidas y enviadas, especialmente en la gestión administrativa o para la realización de estadísticas. Un control sobre los tiempos de respuesta de cada biblioteca contribuye a mejorar el servicio, y conocer los títulos más solicitados ayuda a racionalizar la colección.

Los sistemas de automatización de procesos de PI se pueden agrupar en:

- Sistemas ligados a los catálogos colectivos. En España, ninguno de los catálogos colectivos descritos ha adaptado un programa o módulo de PI a diferencia de lo que ha sucedido en otros países como Estados Unidos con ILLiad de OCLC, Italia con Nilde Network Inter-Library Document Exchange (Mangiaracina et al., 2008) o Alemania con Subito. Estos dos últimos casos han desbancado a la British Library como proveedor internacional en la mayoría de las bibliotecas españolas por su buen servicio y menor coste. http://www.oclc.org/illiad

https://nilde.bo.cnr.it http://www.subito-doc.de

- Sistemas ligados al catálogo local. Un ejemplo es el módulo de PI del sistema de gestión de bibliotecas Millenium usado en la Universidad de Cádiz.

- Programas independientes del catálogo. Aplicaciones propias como es el caso de la red de bibliotecas del CSIC, la Universidad Complutense de Madrid o la Universidad de Extremadura, y los programas comerciales como GTBibSOD.

\section{De DBase a GTBib}

DBase fue el primer sistema de gestión de bases de datos que apareció para micrordenadores, comercializado por la empresa Ashton-Tate a mediados de los 80. Su popular versión Dbase III+ se empleó para la automatización de numerosos procesos en bibliotecas americanas (Eichelberger, 1987), entre ellos el PI (Lingle; Malcom, 1989).

En España también se iniciaron varios proyectos de automatización de PI con Dbase o derivados, como el de la biblioteca de la Universidad de Santiago (AllerÁlvarez, 1992), el proyecto G-Fax (González-Borrajo et al., 1997) o el proyecto SOD de la biblioteca de la Universitat Politècnica de Catalunya (Rodríguez-Gairín, 1996).

Dbase no supo adaptarse a tiempo a la aparición de Windows y fue superado por otros productos como Clipper. Del mismo modo, sólo SOD supo adaptarse a los cambios y con gran visión de futuro fue rediseñado en $\mathrm{Vi}$ sual Objects para trabajar como CGI en un entorno cliente/ servidor sin pasar por ninguna versión Windows. En 2004 y bajo el nombre GTBib se rediseñó por tercera vez en un entorno PHP/MySQL. En la actualidad, GTBib tiene una cuota de mercado de más del $95 \%$ en las universidades españolas.

\section{Gestión de los derechos de autor}

Uno de los principales problemas del servicio de PI en la actualidad es la gestión de los derechos de autor. En el caso de los documentos electrónicos, muchas editoriales los excluyen directamente del $\mathrm{PI}$, otras imponen limitaciones temporales de uso o control del número de accesos al documento, y aún otras exigen que se entreguen siempre en formato impreso o que se eliminen los elementos multimedia de los documentos, obligando a imprimirlos y volverlos a generar en formato imagen antes de utilizarlos para PI.

No es objetivo de este trabajo analizar los aspectos legales ni repercusiones que implica la gestión de derechos de autor. Pero si hablamos de automatización no podemos dejar de mencionar el DRM - digital rights management-, un conjunto de tecnologías que limitan el uso de medios en dispositivos digitales, haciendo que, por ejemplo, sólo puedan utilizarse en un número concreto de dispositivos, abrirse un número de veces o que se bloqueen pasado un período de tiempo concreto.

DRM está ampliamente difundido en música o películas y en nuestro caso afecta básicamente a los documentos en formato pdf. DRM ha sido adoptado plenamente por la British Library (Braid, 2004). Sirva como ejemplo que el 60\% de los documentos enviados por esta biblioteca en agosto de 2010 lo fueron en el formato FileOpen (Madden, 2010), una aplicación DRM totalmente compatible con Acrobat Reader. Este hecho ha sido objeto de amplias críticas y controver-

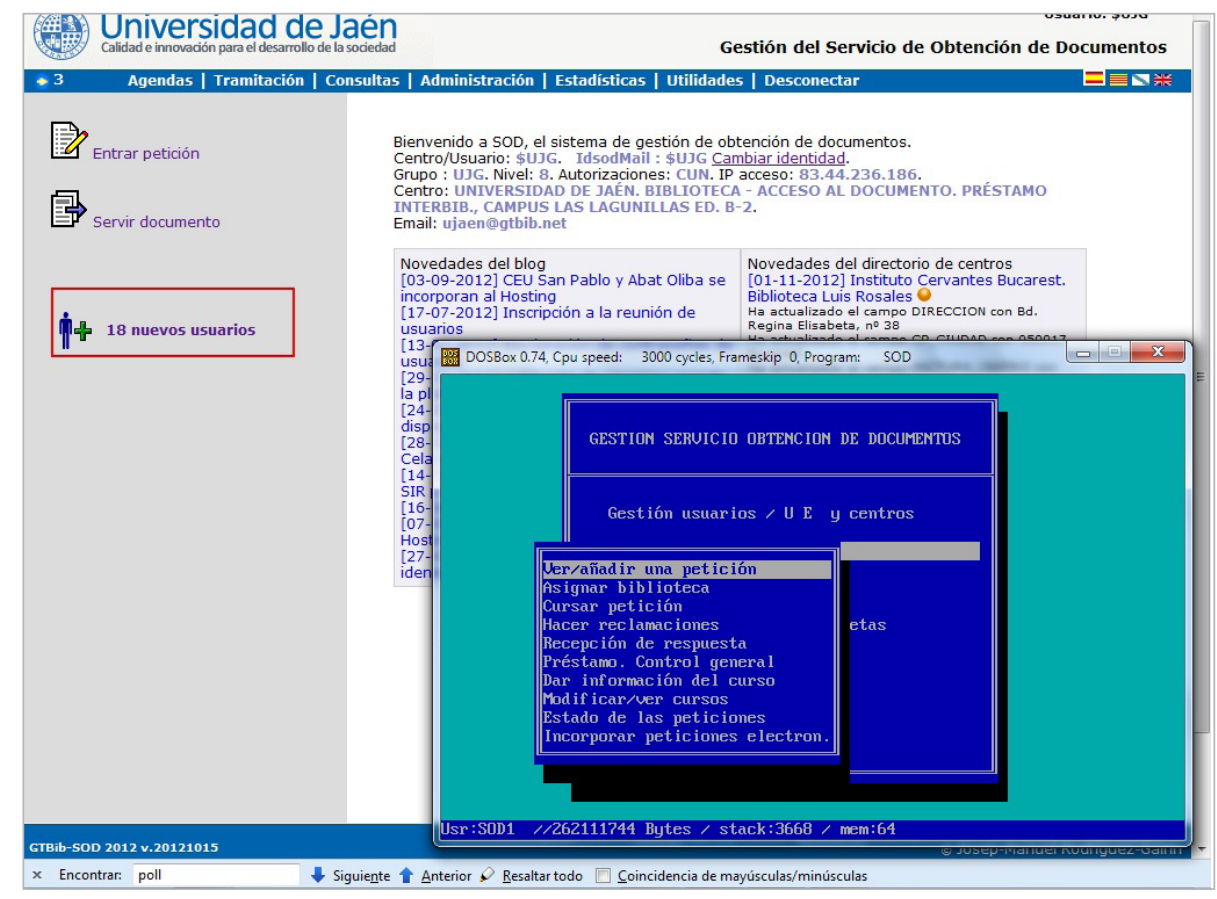

Figura 3. De SOD 93 a GTBib-SOD 2012 
sias (Murray-Rust, 2010), pero la evidencia es que tanto el FileOpen como Adobe Digital Editions están plenamente vigentes en las transacciones de PI de muchas bibliotecas. Esto supone un reto para las aplicaciones de $\mathrm{PI}$, que deberán incorporar módulos para la gestión de estos aspectos. En este sentido podemos mencionar la opción hard copy de la red Nilde citada anteriormente, que convierte un documento pdf estándar en un documento pdf imagen antes de ser enviado al solicitante adaptándolo así a los requerimientos de algunos editores.

http://www.fileopen.com

http://www.adobe.com/es/products/digitaleditions

Aunque el PI de libros electrónicos no está plenamente definido, podemos mencionar proyectos como el del Instituto Cervantes, que contempla incluirlo en su servicio de préstamo de libros electrónicos gestionado con DRM.

http://www.cervantes.es/bibliotecas_documentacion_ espanol/recursos_en_linea/libros_electronicos.htm

\section{Conclusiones}

La automatización de los servicios de PI en las universidades españolas ha seguido un modelo descentralizado en el que cada centro tiene su propio sistema de gestión, a diferencia de lo que ha ocurrido en otros países como Estados Unidos en el que el programa de gestión está centralizado y ligado al catálogo colectivo OCLC. Este aspecto hace que cobren especial importancia las comunicaciones y los estándares para poder interconectar diferentes sistemas.

En tiempos de crisis, en los que la racionalización de las colecciones y los ajustes presupuestarios están a la orden del día, servicios como el PI han sabido aprovechar las nuevas tecnologías y adaptarse a los cambios. Esto justifica su permanencia en un momento en que los profesionales que no trabajan directamente en él pueden pensar que es un servicio en extinción.

Las soluciones de pago por artículo adoptadas en muchos portales de editoriales conocidas como CAS-IAS -Current Awareness Service Individual Article Supply- no siempre son válidas en entornos universitarios que no permiten el pago directo con tarjeta de crédito y cuyos costes deben imputarse a proyectos. En este sentido los servicios de PI adquieren el papel de intermediarios en la transacción.

En definitiva, en la actualidad estamos en un momento en que si sabemos identificar las nuevas necesidades y aprovechar los recursos y avances tecnológicos conseguiremos que servicios como el acceso al documento sigan siendo piezas fundamentales de las nuevas bibliotecas.

\section{Bibliografía}

Aller-Álvarez, Miguel-Ángel. "La informatización del servicio de acceso al documento en la Biblioteca Universitaria de Santiago de Compostela: bases para un primer proyecto". Boletín de la Anabad, 1992, v. 42, n. 2, pp. 55-77. http://dialnet.unirioja.es/servlet/fichero_articulo?codigo $=2$ 24180\&orden $=57565$

Anglada, Lluís. "Collaborations and alliances: social intelligence applied to academic libraries". Library management, 2007 , v. 28 , n. $6 / 7$, pp. 406-415. http://dx.doi.org/10.1108/01435120710774530

Angós-Ullate, José-María; Fernández-Ruiz, María-Jesús; Salvador-Oliván, José-Antonio. "Intercambio de documentos electrónicos en internet usando Prospero". En: 8as Jornadas españolas de documentación (Fesabid 2003), 2003, pp. 283-293.

Baiget, Tomàs. "Números nacionales de X.28 y servicio X.32". El profesional de la información, 1992, n. 6.

http://www.elprofesionaldelainformacion.com/ contenidos/1992/julio/nmeros_nacionales_de_x28_y_ servicio_x32.html

Bartolo, Laura M. "Automated ILL analysis and collection development: a hi-tech marriage of convenience". Library acquisitions: practice \& theory, 1989, v. 13, n. 4, pp. 361367.

http://dx.doi.org/10.1016/0364-6408(89)90047-1

Braid, Andrew. "The use of a digital rights management system in a document supply service". Interlending and document supply, 2004, v. 32, n. 3, pp. 189-191.

http://dx.doi.org/10.1108/02641610410699759

Breeding, Marshall. "Looking forward to the next generation of discovery services". Computers in libraries, 2012, v. 32, n. 2, pp. 28-31.

http://cat.inist.fr/?aModele $=$ afficheN\&cpsidt $=25639812$

Casares, Laia; Edo, Montserrat; Olivé, Montserrat; Rodríguez-Gairín, Josep-Manuel. "Projectes de cooperació en matèria de préstec interbibliotecari dins el marc del Consorci de Biblioteques Universitàries de Catalunya." En: 6es Jornades catalanes de documentació, 1997, pp. 213-223. http://www.cbuc.cat/cbuc/content/download/3289/19793/ version/3/file/11projectes_casares.pdf

Duarte, Gema; Olivé, Montserrat. "Una experiencia práctica del correo electrónico en el servicio de obtención de documentos de la Universitat Rovira i Virgili". En: V Jornadas españolas de documentación automatizada, 1996, pp. 859863.

Eichelberger, Susan. "Using dBase III in the library". Library software review, 1987, v. 6, n. 4, pp. 178-89.

González-Borrajo, José-María; Lomba-Otero, Saúl; PérezRodríguez, Marcos; Tumbo-Guerner, Francisco-Javier. "GFax". En: II Xornadas de arquivos, bibliotecas e museos de Galicia, 1997, pp. 339-348.

Gould, Sara. "Sending ILL requests by e-mail: a discussion and IFLA guidelines". Interlending \& document supply, 2000, v. 28 , n. 2, pp. 73-78.

http://dx.doi.org/10.1108/02641610010331525

ISO. ISO 10160:1997. Information and documentation -Open systems interconnection - Interlibrary loan application service definition, 1997, $58 \mathrm{pp}$.

http://www.iso.org/iso/catalogue_detail?csnumber $=22247$

ISO. ISO 10161-1:1997. Information and documentation -Open systems ilnterconnection - Interlibrary loan application protocol specification - Part 1: Protocol specification, 1997, $109 \mathrm{pp}$.

http://www.iso.org/iso/catalogue_detail?csnumber=22248 
ISO. ISO 10161-2:1997. Information and documentation -Open systems interconnection - Interlibrary loan application protocol specification - Part 2: Protocol implementation conformance statement (PICS) proforma, 1997, $38 \mathrm{pp}$.

http://www.iso.org/iso/home/store/catalogue_tc/ catalogue_detail.htm?csnumber=21389

ISO. ISO 23950:1998. Information and documentation -- Information retrieval (Z39.50) - Application service definition and protocol specification, 1998, $154 \mathrm{pp}$.

http://www.iso.org/iso/catalogue_detail.htm?csnumber=27446

Jaramillo, George R.; Squire, Jan S. "Uncover-instant article access". Serials review, 1990, v. 16, n. 3, pp. 29-37. http://dx.doi.org/10.1016/S0098-7913(05)80004-8

Landes, Sonja. "Ariel document delivery: a cost-effective alternative to fax". Interlending and document supply, 1997, v. 25, n. 3, pp. 113-117.

http://dx.doi.org/10.1108/02641619710170519

Leeves, Juliet. "Automation of ILL management systems". Interlending and document supply, 1993, v. 21, n. 3, pp. 12-17. http://dx.doi.org/10.1108/02641619310154656

Lieberthal, Susan P. "Management software for interlibrary loans: pathways to becoming a high performing interlibrary loan department". Journal of interlibrary loan, document delivery \& information supply, 2000, v. 11, n. 2, pp. 5-22. http://dx.doi.org/10.1300/J110v11n02_02

Lingle, Virginia A.; Malcom, Dorothy L. "Interlibrary loan management with microcomputers: a descriptive comparison of software". Medical reference services quarterly, 1989 , v. 8, n. 2, pp. 41-64.

http://dx.doi.org/10.1300/J115v08n02_04

López-Manzanedo, María-Jesús; Vázquez-Valero, Manuela; Goas-Paz, Ana. El préstamo interbibliotecario en España. Madrid: Subdirección General de Coordinación Bibliotecaria, 1996. ISBN: 8481811378

Luther, Judy; Kelly, Maureen C. "The next generation of discovery". Library journal, 15 marzo 2011.

http://www.libraryjournal.com/lj/home/889250-264/the next_generation_of_discovery.html.csp

Madden, Kate. “FileOpen - The British Library's preferred DRM service", 2010.

http://www.bl.uk/reshelp/atyourdesk/docsupply/help/ receiving/deliveryoptions/electronic/fileopen/index.html

Mangiaracina, Silvana; Zaetta, Marta; De-Matteis, Daniele; Tugnoli, Alessandro; Beghelli, Enrico; Tenaglia, Giacomo. "Nilde: developing a new generation tool for document delivery in Italy". Interlending and document supply, 2008, v. 36, n. 3, pp. 167-177.

http://dx.doi.org/10.1108/02641610810897908

Méndez-Martínez, Carmen; Ruz-Baños, Miguel; Aldehuela-Serra, María-Carmen. "Perspectivas del servicio de préstamo interbibliotecario en las bibliotecas públicas españolas". En: $2^{\circ}$ Congreso nacional de bibliotecas públicas, 2004, pp. 425-433.

http://hdl.handle.net/10421/795

Merlo-Vega, José-Antonio. “Cooperación en las bibliotecas universitarias: fundamentos y redes cooperativas". Boletín de la Asociación Andaluza de Bibliotecarios, 1999, v. 54, pp. 33-57.

http://gredos.usal.es/jspui/bitstream/10366/18000/1/ $D B D \_C o o p$. btcas. univ..pdf

Merlo-Vega, José-Antonio; Sorli-Rojo, Ángela. "La cooperación bibliotecaria en tiempos de Internet". Anuario Socadi de información y documentación, 1998, pp. 245-254.

http://gredos.usal.es/jspui/handle/10366/17989

Merlo-Vega, José-Antonio; Sorli-Rojo, Ángela. "Bases de datos de sumarios de publicaciones periódicas realizadas por bibliotecas españolas". Revista española de documentación científica, 2002, v. 25, n. 2, pp. 195-202. http://hdl.handle.net/10261/9450

Merry, Karen. "Adonis: a new era in document delivery". Interlending and document supply, 1988, v. 16, n. 2, pp. 65-69. http://dx.doi.org/10.1108/eb008564

Murray-Rust, Peter. Digital rights management on electronic interlibrary loans: a freedom of information request to The British Library, 2010.

http://www.whatdotheyknow.com/request/digital_rights_ management_on_ele

Nagelsmeier-Linke, Marlene. "Automatisierte fernleihe und elektronische dokumentlieferung in den bibliotheken. Der stand der entwicklung". Libri, 1994, v. 44, n. 4, pp. 348-368. http://dx.doi.org/10.1515/libr.1994.44.4.348

NISO. ANSI/NISO Z39.88 - 2004 (R2010). The OpenURL framework for context-sensitive services, 2004, ISBN: 9781 880124611

http://www.niso.org/apps/group_public/project/details. php?project_id $=82$

Navarrete-Cortés, José; Navarrete-Cortés, Fernando. "Sad@Exe: una interface para el envío de peticiones de préstamo interbibliotecario y búsquedas bibliográficas mediante correo electrónico". Boletín de la Asociación Andaluza de Bibliotecarios, 2000, v. 15, n. 59, pp. 15-22.

http://www.aab.es/aab/index.php?option=com_content\&t ask=view\&id $=75 \& /$ temid $=64$

Porat, Lynne. "Automation of interlibrary loan services: effects on the patron and the library". Interlending and document supply, 2001, v. 29, n. 3, pp. 108-113. http://dx.doi.org/10.1108/02641610110400293

Ramos, Inmaculada; Montes, Ángel. "Suministro electronico de documentos via internet: Ariel". El profesional de la información, 1998, v. 7, n. 10, pp. 28-30.

http://www.elprofesionaldelainformacion.com/ contenidos/1998/octubre/suministro_electronico_de_ documentos_via_internet_ariel.html

Rodríguez-Gairín, Josep-Manuel. "Noves perspectives en l'accés al document original." Item, 1996, v. 18, pp. 17-27. http://www.raco.cat/index.php/Item/article/view/22472/22306

Rodríguez-Gairín, Josep-Manuel. "Winsumar: distribución y consulta electrónica de sumarios de revistas". En: // Jornadas de bibliotecas digitales, 2001. 
http://infonautica.net/docs/jbidi/jbidi2001/12_2001.pdf

Rodríguez-Gairín, Josep-Manuel; Somoza-Fernández, Marta. "Los agentes de software desde la perspectiva bibliotecaria: delegación de tareas en el préstamo". En: 7o Congreso del capítulo español de ISKO: La dimensión del conocimiento, 2005, pp. 521-533.

http://dialnet.unirioja.es/servlet/articulo?codigo $=2969258$

Schnell, Eric H. "Freeing Ariel: the Prospero electronic document delivery project". Journal of interlibrary loan, document delivery and information supply, 2000, v. 10, n. 2, pp. 89-100.

http://dx.doi.org/10.1300/J110v10n02_08
Stein, Joan E. IFLA Guidelines for best practice in interlibrary loan and document delivery, 2007.

http://www.ifla.org/files/docdel/documents/guidelinesbest-practice-ill-dd-en.pdf

Urbano, Cristóbal. "Obtención de documentos: una lectura de las últimas revisiones bibliográficas desde la situación española". Anuario Socadi de información y documentación, 1997, pp. 191-201.

Vázquez-Valero, Manuela; Román-Román, Adelaida. "El sistema nacional de préstamo, una necesidad urgente". Boletín de la Anabad, 1986, v. 36, n. 4, pp. 803-809.

http://dialnet.unirioja.es/servlet/articulo?codigo $=803905$

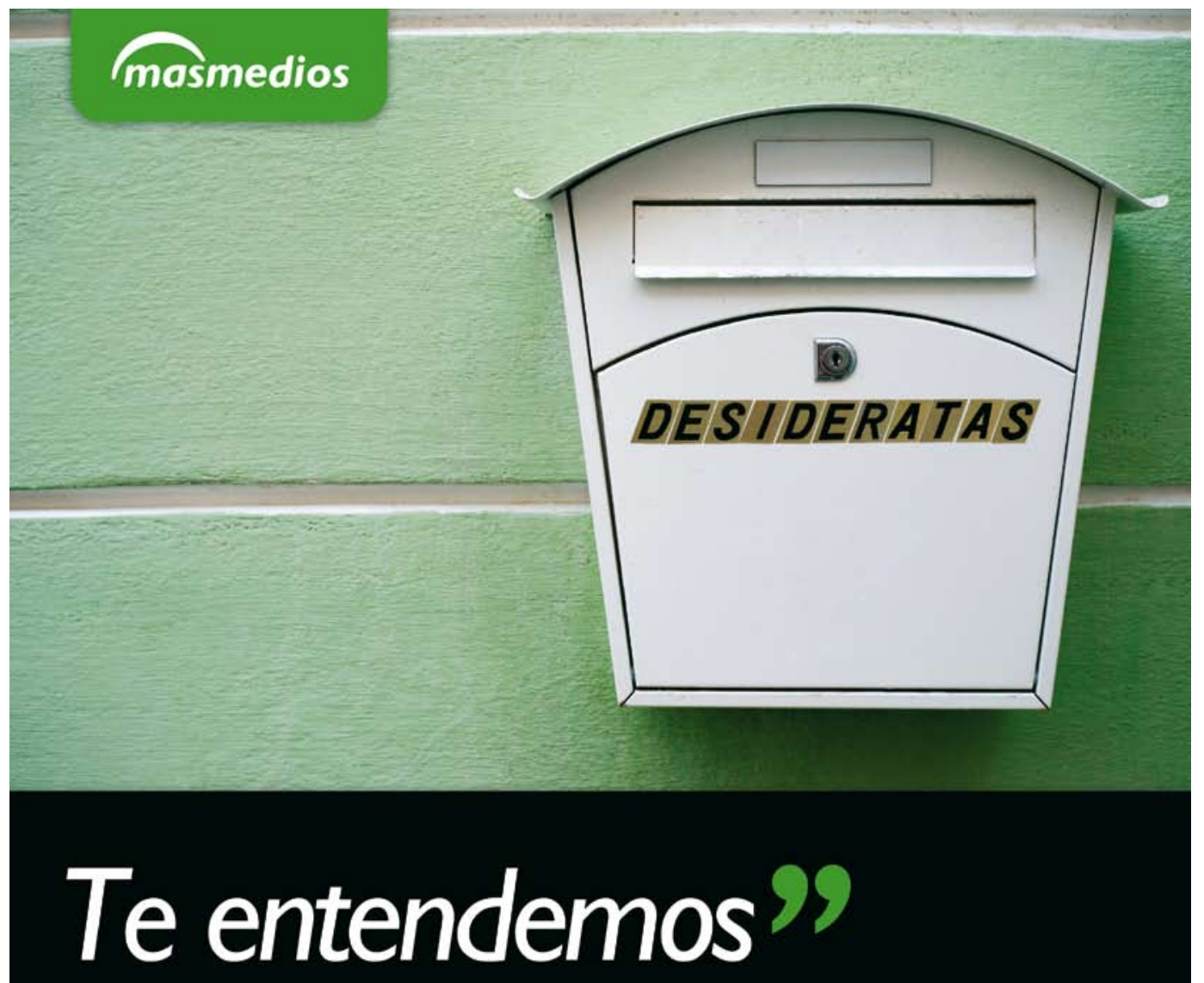

Sistemas de gestión de bibliotecas Open Source Interfaces interactivas y OPACs

Repositorios OAl

Gestión documental y de archivos

Digitalización

Outsourcing de servicios documentales

Desarrollo de sitios web / multimedia / e-learning

Comunicación y e-marketing de servicios de información
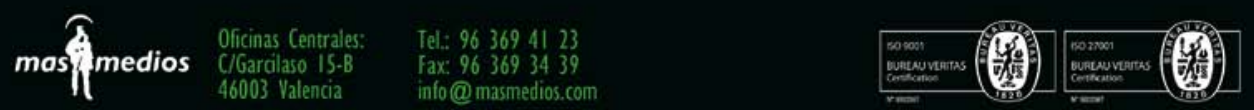

www. masmedios.com 